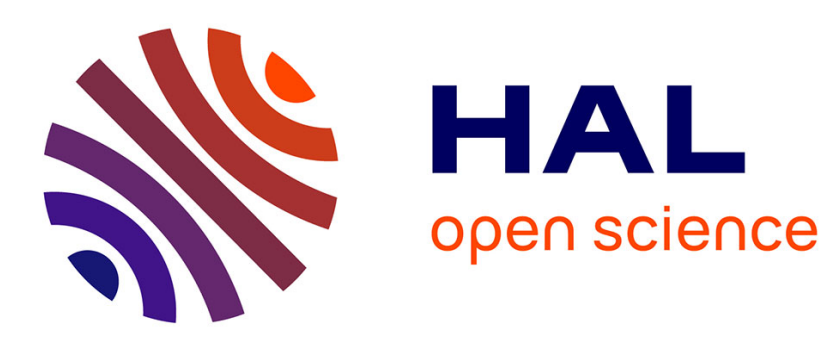

\title{
Design of Abiological Digital Poly(phosphodiester)s
}

Laurence Charles, Jean-Francois Lutz

\section{To cite this version:}

Laurence Charles, Jean-Francois Lutz. Design of Abiological Digital Poly(phosphodiester)s. Accounts of Chemical Research, 2021, 54 (7), pp.1791-1800. 10.1021/acs.accounts.1c00038 . hal-03454786

\section{HAL Id: hal-03454786 \\ https://hal.science/hal-03454786}

Submitted on 29 Nov 2021

HAL is a multi-disciplinary open access archive for the deposit and dissemination of scientific research documents, whether they are published or not. The documents may come from teaching and research institutions in France or abroad, or from public or private research centers.
L'archive ouverte pluridisciplinaire $\mathbf{H A L}$, est destinée au dépôt et à la diffusion de documents scientifiques de niveau recherche, publiés ou non, émanant des établissements d'enseignement et de recherche français ou étrangers, des laboratoires publics ou privés. 


\section{Design of Abiological Digital Poly(phosphodiester)s}

Laurence Charles ${ }^{* 1}$ and Jean-François Lutz*2

${ }^{1}$ Aix Marseille Université, CNRS, Institute for Radical Chemistry, UMR 7273, 23 Av Escadrille Nomandie-Niemen, 13397 Marseille Cedex 20, France.

E-mail: laurence.charles@univ-amu.fr

${ }^{2}$ Université de Strasbourg, CNRS, Institut Charles Sadron UPR22, 23 rue du Loess, 67034 Strasbourg Cedex 2, France.

Jean-François Lutz: jflutz@,unistra.fr

Published in Accounts of Chemical Research, 54, 1791-1800 (2021)

https://pubs.acs.org/doi/10.1021/acs.accounts.1c00038 


\section{CONSPECTUS.}

In biological systems, the storage and transfer of genetic information rely on sequencecontrolled nucleic acids such as DNA and RNA. It has been realized for quite some time that this property is not only crucial for Life, but could also be very useful in Human applications. For instance, DNA has been actively investigated as a digital storage medium over the last decade. Indeed, the "hard-disk of Life" is an obvious choice and a highly-optimized material for storing data. Through decades of nucleic acids research, technological tools for parallel synthesis and sequencing of DNA are readily available. Consequently, it has already been demonstrated that different types of documents (e.g. texts, images, videos, industrial data) can be stored in chemically-synthesized DNA libraries. Yet, DNA is subject to biological constraints and its molecular structure cannot be easily varied to match technological needs.

In fact, DNA is not the only macromolecule that enables data storage. In recent years, it has been demonstrated that a wide variety of synthetic polymers can also be used for such a purpose. Indeed, modern polymer synthesis allows preparation of synthetic macromolecules with precisely-controlled monomer sequences. Altogether, about a dozens of synthetic digital polymers have already been described and many more can be foreseen. Among them, sequence-defined poly(phosphodiester)s are one of the most promising options. These polymers are prepared by stepwise phosphoramidite chemistry like chemically-synthesized oligonucleotides. However, they are constructed with non-natural building blocks and therefore share almost no structural characteristics with nucleic acids, except phosphate repeat units. Still, they contain readable digital messages that can be deciphered by nanopore sequencing or mass spectrometry sequencing.

In this Account, we describe our recent research efforts for synthesizing and sequencing optimal abiological digital poly(phosphodiester)s. A major advantage of these polymers over DNA is that their molecular structure can easily be varied to tune their properties. During the 
last five years, we have engineered the molecular structure of these polymers to adjust crucial parameters such as storage density, storage capacity, erasability and readability. Consequently, high-capacity PPDE chains, containing hundreds of bits per chains, can now be synthesized and efficiently sequenced using a routine tandem mass spectrometer. Furthermore, sequencing data can be automatically decrypted with the help of a decoding software. This new type of coded matter can also be edited using practical physical triggers such as light and organized in space by programmed self-assembly. All these recent improvements are summarized and discussed herein. 


\section{KEY REFERENCES}

- Al Ouahabi, A.; Charles, L.; Lutz, J.-F. Synthesis of Non-Natural Sequence-Encoded Polymers Using Phosphoramidite Chemistry. J. Am. Chem. Soc. 2015, 137, 5629-5635. ${ }^{1}$ This publication describes the first example of synthesis of digitally-encoded PPDE, which are not nucleic acids. Sequencing was not addressed in this first publication.

- Al Ouahabi, A.; Amalian, J.-A.; Charles, L.; Lutz, J.-F. Mass spectrometry sequencing of long digital polymers facilitated by programmed inter-byte fragmentation. Nat. Commun. 2017, 8,967. ${ }^{2}$ In general, mass spectrometry is a limited technique to sequence DNA and non-natural PPDE. This article describes for the first time that long PPDE chains with an optimized molecular design can be efficiently sequenced in a routine mass spectrometer.

- König, N. F.; Al Ouahabi, A.; Oswald, L.; Szweda, R.; Charles, L.; Lutz, J.-F. Photoeditable macromolecular information. Nat. Commun. 2019, 10, 3774. ${ }^{3}$ It was shown in this article that digital sequences of PPDE can be edited by UV light. For instance, light irradiation can be used to erase, reveal of modify an information sequence.

- Laurent, E.; Amalian, J.-A.; Parmentier, M.; Oswald, L.; Al Ouahabi, A.; Dufour, F.; Launay, K.; Clément, J.-L.; Gigmes, D.; Delsuc, M.-A.; Charles, L.; Lutz, J.-F. High-Capacity Digital Polymers: Storing Images in Single Molecules. Macromolecules 2020, 53, 4022-4029. ${ }^{4}$ In this publication, expanded monomer alphabets based on 4 or 8 symbols were used to encode digital PPDE. The alphabet with 8 symbols allows a storage density that is higher than in DNA. 


\section{INTRODUCTION.}

In 2013, an Account entitled "Writing on Polymer Chains" was published in this journal. ${ }^{5}$ It explains that sequence-controlled copolymerizations can be used as a tool to write intentional information in the microstructure of synthetic polymers. However, at that time, the polymerization approach that was used was still rather uncontrolled and was therefore compared to an imperfect handwriting. Thus, the conspectus image of that earlier paper was questioning if a more precise molecular "typesetting" would ever be attained. Seven years later, this new Account shows that it has now become true. Indeed, the field of sequence-controlled polymers has drastically improved over the last few years. ${ }^{6-8}$ For instance, the first example of a non-biological digital polymer was reported in $2014,{ }^{9}$ followed by several other examples. ${ }^{1,10-}$

${ }^{16}$ In such polymers, binary encryption is obtained by using coding comonomers that are assembled into a defined information sequence. ${ }^{17,18}$ Of course, the binary code is not the only language that can be used to encode a polymer chain and other types of informational polymers exist. ${ }^{19}$ Overall, this emerging domain is not only an academic issue but has also opened up new applications areas for synthetic polymers; for example, in the fields of data storage, longterm storage, materials traceability, plastics recycling, cryptography and anti-counterfeiting technologies. Despite the recentness of this research area, several information-containing polymers have been patented and some of them are already commercialized as chemical taggants.

In order to be functional, an informational polymer must have, at least, the two following features: (i) a writing chemistry that enables chain encryption, (ii) a reading analytical technique that permits to recover information. Other properties such as erasing, rewriting, copying and editing are desired but not strictly mandatory for the applications listed above. In terms of polymer chemistry, all abiological informational polymers are synthesized by multistep growth synthesis; a mechanism in which coded building blocks are assembled one- 
by-one to a growing chain. ${ }^{20}$ Such chemistry is performed in most cases on solid supports, even though examples of synthesis on soluble supports or even in solution have been reported. ${ }^{18}$ Regarding sequencing, the range of analytical techniques that can decode synthetic polymers is not yet as broad as for nucleic acids and proteins. ${ }^{21,22}$ So far, only two main analytical tools have been validated for abiological polymer sequencing, namely mass spectrometry ${ }^{23}$ and nanopore analysis. ${ }^{24}$

Among all the types of informational polymers that have been reported, some categories seem more promising than others. For instance, non-natural PPDE have experienced rapid progress during the last few years. As mentioned in the Conspectus, these polymers are synthesized by solid-phase iterative phosphoramidite chemistry; a technique that has been historically developped for the chemical-synthesis of DNA. ${ }^{25}$ Still, the phosphoramidite approach is not restricted to nucleoside monomers and a wide variety of non-natural building blocks can also be employed in this technique. ${ }^{26,27}$ In fact, when all building blocks are nonnatural, phosphoramidite chemistry is no longer a biochemical technique but simply a polymer chemistry tool. Thus, in this Account, we describe how this chemistry can be efficiently utilized for the synthesis of digital polymers. In addition, our recent accomplishments for decoding these polymers by mass spectrometry are summarized. Generally speaking, mass spectrometry is not a technique of choice for the sequencing of natural poly(phosphodiester)s such as DNA and RNA. ${ }^{28}$ Indeed, uncontrolled fragmentations and resolution problems render this technique poorly-effective for nucleic acids sequencing. However, with careful polymer design and methodological improvements, we have shown that this situation can be drastically different for non-natural PPDE. These significant advances are summarized and commented within the next pages. 


\section{PHOSPHORAMIDITE POLYMER CHEMISTRY.}

Digital PPDE are synthesized by PPC. As mentioned in the introduction, phosphoramidite chemistry has been first introduced for the synthesis of sequence-defined oligonucleotides ${ }^{29}$ It is a multistep solid-phase approach employing monomers containing a reactive phosphoramidite group and a protected alcohol (Figure 1a). The protecting group is usually the acid labile DMT group but it can also be a light-sensitive moiety such as NPPOC (Figure 1b).$^{30,31}$ The process is performed on a $\mathrm{OH}$-functional solid support and relies on a repeated cycle of three or four reactions: (i) coupling of the phosphoramidite moiety of the monomer to the $\mathrm{OH}$ of the support, (ii) deactivation of the unreacted alcohols with a capping agent, (iii) oxidation of the formed phosphite linkage into a phosphate, (iv) removal of the OH-protecting group to enable the installation of the next monomer. Only steps (i), (iii) and (iv) are mandatory, whereas step (ii) can be avoided for very short sequences but is recommended for longer ones. This chemistry is highly-optimized, highly efficient and can be performed on automated DNA synthesizers. ${ }^{32}$ Furthermore, both biological and non-biological monomers can be used in this technique. Although synthetic monomers were initially mainly developped to incorporate nonnatural motifs in nucleic acids, they later have been used for the synthesis of bio-hybrid structures (i.e. DNA-polymer conjugates) $)^{33,34}$ as well as fully synthetic oligomers and polymers. ${ }^{1,26,27,35}$ For instance, PPC was used to synthesize all the polymers that are discussed in this Account. Short sequences are usually synthesized manually on crosslinked polystyrene resins, ${ }^{1,31}$ whereas longer polymers are prepared on DNA synthesizers. ${ }^{36}$ In the latter case, since the number of reservoirs is often conceived for only four nucleotides, some syntheses imply bottle exchanges. Nevertheless, automated PPC is a robust technique. Coupling yields for nonnatural monomers are often above $99 \%$ and therefore synthetic polymers with chain-lengths above DP100 can be prepared. ${ }^{36}$ Furthermore, when more than one monomer is used, this 
multistep method allows synthesis of uniform sequence-defined polymers. ${ }^{6}$ Hence, messages can be written on synthetic PPDE using preset monomer alphabets. ${ }^{19}$

\section{CODING MONOMER ALPHABETS.}

As summarized in an earlier perspective article, ${ }^{19}$ in order to write information on a polymer, it is first mandatory to define writing conventions. These rules define for example the number of symbols (i.e. coded monomers) and the writing direction (i.e. chain directionality) but may also include other information such as prefix addresses, document format, cryptographic keys, corrections codes or compressions codes. As in any language, the reader should know some basic rules to understand a message. For instance, to write digital information on a polymer, molecular bits shall be defined. Figure 1 display some coded monomers that we have studied over the last years. In our initial work, an alphabet based on two symbols was used. ${ }^{1}$ Indeed, two monomers of different molecular structure can symbolize a bit-0 and a bit-1 (i.e. storage density of one bit/monomer). ${ }^{17}$ For example, $\mathbf{M}_{\mathbf{1}}$ and $\mathbf{M}_{\mathbf{3}}$ have a different molar mass and can therefore be distinguished from another by mass spectrometry. Thus, ordered sequence of these two monomers can potentially be decrypted by MS/MS ${ }^{37}$ Our laboratory convention is that the monomer with the lightest and heaviest molecular weights symbolize bits 0 and 1, respectively. Yet, as pointed out in the early days of this research, ${ }^{19}$ binary encryption can also be achieved using more symbols than two. For instance, the use of four different symbols such as monomers M1-M4 enables to encode binary dyads 00, 01, 10 and 11 (i.e. storage density of 2 bits/monomer). ${ }^{4}$ The use of eight symbols such as $\mathbf{M}_{\mathbf{1}}-\mathbf{M}_{\mathbf{8}}$ allows encryption of triads 000, 001, 010, 011, 100, 101, 110 and 111 (i.e. storage density of 3 bits/monomer). ${ }^{4}$ This strategy can potentially be extended to even more symbols, but this was not achieved yet. It shall be mentioned that using more symbols potentially leads to more synthesis and decryption 
problems. For example, bulky monomers such as $\mathbf{M}_{7}$ and $\mathbf{M}_{8}$ require optimized coupling conditions to be incorporated in high yields.

Monomers M1-M8 are appropriate for MS/MS sequencing but other monomer combinations may be useful for other sequencing techniques. ${ }^{21}$ Indeed, other properties than mass differences can be exploited to design decodable digital polymers. For example, $\mathbf{M}_{\mathbf{1}}$ and M9 have different bulkiness and can be used as molecular bits for nanopore sequencing. ${ }^{38}$ Properties such as molar mass, bulkiness or polarity can be set from premade monomers but can also be adjusted after polymer synthesis using chemical or physical modifications. For example, monomers $\mathbf{M}_{9}$ and $\mathbf{M}_{10}$ can be modified by post-polymerization orthogonal CuAAC. ${ }^{39}$ Monomers M11-M14 carry photo-sensitive or photo-inert substituents and their coded sequences can be adjusted by light, as described in a following section.

\section{INITIAL ATTEMPTS OF DIGITAL DPPE SEQUENCING.}

Although various analytical methods can be used for the sequencing of nucleic acids and polymer-DNA bio-hybrids, ${ }^{22,40,41}$ only a few of them allows decoding of synthetic digital polymers. ${ }^{21}$ The sequencing of information-containing PPDE has been investigated so far using two main techniques, which are nanopore sequencing and MS/MS. Still, the former method is for the moment much less explored than the latter. In nanopore sequencing, polyelectrolytes are subjected to an ionic current and thread through holes of defined size, which can be poreforming proteins or solid-state pores. ${ }^{24}$ The interactions of the monomer units with the pore lead to voltage variations that can be recorded and eventually related to a sequence. PPDE, which contain repeat phosphate units, are polyanions that can be analyzed by this technique. In a first study, homopolymers of $\mathbf{M}_{1}$ were analyzed in either $\alpha$-hemolysin or aerolysin nanopores. ${ }^{42}$ It was concluded that the high flexibility of these chains hinders their efficient translocation and that an optimization of their molecular structure might be mandatory to 
overcome the entropic barrier. More recently, bio-hybrid macromolecular analytes containing abiotic inner sequences flanked by di-deoxyadenosine extremities were analyzed in aerolysin mutants. ${ }^{38}$ Digitally-encoded oligomers based on $\mathbf{M}_{\mathbf{1}}$ and $\mathbf{M} \mathbf{9}$ and containing from 1 to 4 bits of information were sequenced with single-bit resolution. However, the translocation of a given sequence generates several types of events and machine learning is mandatory to interpret these signals.

The MS/MS sequencing of sequence-coded PPDE was at first also not straightforward. Most of our research has been performed using ESI in the negative ion mode to enable deprotonation of PPDE. When subjected to CID, polymers undergo fragmentations, which may be correlated to a sequence. ${ }^{21,23}$ Although main-chain fragmentations are wished, side-chain fragmentations may result in information loss that prevents sequencing. In the case of PPDE, cleavage of main-chain phosphate bonds is induced by CID. ${ }^{37}$ Thus, short digitally-encoded PPDE can be deciphered by MS/MS. In that regard, synthetic PPDE are more convenient for MS/MS than DNA, which usually leads to unwanted side-chain fragmentation leading to loss of nucleobases. ${ }^{43}$ However, the situation becomes less favorable for PPDE of increasing length. ${ }^{37}$ With eight fragments per phosphate group, the total number of ions in CID spectra rapidly increases with the number of monomers, which dilutes the signal of dissociating PPDE species. Consequently, for very long digital sequences, some sequencing fragments are hardly detected and sequence determination becomes more difficult. In fact, the upper chain-length for de novo sequencing of PPDE was estimated to be at DP50. Beyond that limit, full sequence coverage is no longer achieved by MS/MS. Still, one major advantage of synthetic informational polymers is the fact that their molecular structure can be tuned to optimize their properties, including their MS/MS readability. ${ }^{19}$ Based on these initial results, strategies for improving MS/MS sequencing were conceived. They are discussed in the next section. 


\section{IMPROVED POLYMER DESIGN FOR OPTIMAL MS/MS SEQUENCING.}

As described in the previous section, raw PPDE copolymers can only be sequenced by MS/MS until a maximum chain-length of 50 coded units. Yet, this situation can be drastically improved by polymer design. The starting point of that progress was the observation that main-chain alkoxyamine groups, which include N-O-C bonds with low dissociation energy, ${ }^{44}$ are easily broken upon CID. ${ }^{10}$ Interestingly, when co-existing with main-chain amide ${ }^{10}$ or phosphate ${ }^{12}$ linkages, these moieties are preferentially cleaved. This discovery led us to the design of easilydecodable digital PPDE, which are depicted in Figure 2a. In this strategy, an alkoxyaminecontaining linker (such as $\mathbf{L}_{\mathbf{1}}$ in Figure $2 \mathrm{~b}$ ) is periodically incorporated in a PPDE digital chain. This can be easily achieved by automated PPC by using the corresponding phosphoramidite building block. ${ }^{2}$ This linker is usually included every eight phosphates, ${ }^{2}$ although different periodicities have also been studied. ${ }^{4}$ When subjected to CID, alkoxyamine links are selectively cleaved and therefore the polymer is decomposed into a library of predictable segments, as schematized in Figure 2c. Since the digitally-encoded fragments may have the same comonomer composition and therefore be isobaric, a molecular address is needed to indicate where a specific fragment was positioned in the initial sequence. This was achieved by using tags of specific molar mass and isotopic distribution. ${ }^{2}$ We used for that purpose commercial nucleoside phosphoramidites but synthetic motifs could be, of course, used as well. The tags induce unique mass shifts, which permits unequivocal identification of each fragment. Yet, it shall be specified that the molar mass of these tags shall fulfil stringent requirements to be compatible with the coded alphabet used in the digitally-encoded segments. ${ }^{2}$ The same predictable fragments can also be generated in-source by raising the cone voltage of the mass spectrometer. Doing so, these fragments can then be subjected to further dissociations (in a socalled pseudo-MS ${ }^{3}$ experiment) that lead to phosphate bond cleavage and digital decoding (Figure 2c). Ultimately, the whole information sequence of the initial polymer can be 
reconstructed. This strategy can be performed in a routine mass spectrometer and was used in our initial publication to decrypt digital PPDE chains with a storage capacity as high as 64 bits/chain. ${ }^{2}$ Using expanded alphabets $\mathbf{M}_{1}-\mathbf{M}_{4}$ and $\mathbf{M}_{1}-\mathbf{M}_{\mathbf{8}}$, this concept was later successfully used to decode polymers with chain capacities as high as 144 bits/chain. ${ }^{4}$ However, in these initial works, spectra decryption was performed manually, which is time-consuming and tedious. Indeed, the linker $\mathbf{L}_{1}$ leads to the formation of undesired rearrangements upon activation and therefore to the formation of intense side peaks that prevent automated decoding. Although a software named MS-DECODER was developped in 2017 for the automated decryption of synthetic informational polymers, ${ }^{45}$ it could not be applied to PPDE because of these side peaks. This problem was solved in 2020 using an optimized alkoxyamine linker $\mathbf{L}_{2}$ (also known as RISC2) that prevents side-products formation in CID. ${ }^{46}$ Using this optimal design, MS-DECODER can be used and digitally-encoded PPDE can be automatically decrypted. This recent discovery opens up interesting horizons for the design and sequencing of PPDE of very high storage capacity. For example, Figure 3 shows the encryption and automated decryption of a polymer containing 440 bits of information. ${ }^{47}$

\section{MACROMOLECULAR INFORMATION EDITING.}

Beyond encryption, molecular storage and decryption, synthetic polymer chemistry offers unique possibilities to edit digital information. For instance, we have recently described the synthesis and MS/MS sequencing of light-sensitive PPDE (Figure 4). ${ }^{3}$ Monomers $\mathbf{M}_{11}-\mathbf{M}_{14}$ in Figure 1 enable various possibilities of photo-modification. For example, $\mathbf{M}_{11}$ and $\mathbf{M}_{12}$ have a different molar mass and can therefore be used as a binary alphabet to write digital messages that can be deciphered by MS/MS. Yet, the $o$-nitrobenzyl and $o$-nitroveratryl ether side-chains of $\mathbf{M}_{11}$ and $\mathbf{M}_{12}$ are both cleaved when exposed to irradiation at $\lambda=365 \mathrm{~nm}$, thus leading to a non-coded homopolymer as depicted in Figure 4a. Such a simple light erasure mechanism 
could be very interesting for the development of erasable memories. Figure $4 \mathrm{~b}$ shows a different concept, in which a non-decipherable sequence is revealed by light. Monomers $\mathbf{M}_{11}$ and $\mathbf{M}_{13}$ are isomers and therefore their copolymers cannot be deciphered by MS/MS, even if they contain a defined digital sequence. However, upon irradiation, the $o$-nitrobenzyl sidechains of $\mathbf{M}_{11}$ are selectively cleaved because the $p$-nitrobenzyl ether motifs of $\mathbf{M}_{13}$ are not light sensitive. Hence, after light exposure, the monomers have a different molar mass and their sequence can be deciphered by MS/MS. The combination of $\mathbf{M}_{11}$ and $\mathbf{M}_{13}$ is therefore a kind of invisible binary ink that can be revealed by light. Site-directed light-modifications can also be achieved in digital PPDE synthesized with monomers $\mathbf{M}_{11}, \mathbf{M}_{13}$ and $\mathbf{M}_{14}$. After synthesis, the TIPS protecting groups of $\mathbf{M}_{14}$ are removed, thus leading to a digital polymer, in which $\mathrm{OH}$ side-groups are 0-bits and isomers $\mathbf{M}_{11}$ and $\mathbf{M}_{13}$ are 1-bits. When exposed to light, a $1 \rightarrow 0$ mutation occurs exclusively on $\mathbf{M}_{11}$, as previously explained. As a consequence, the meaning of a digital sequence can be altered by light.

\section{SPATIAL ORGANIZATION OF LIBRARIES OF DIGITAL PPDE.}

When a digital polymer is synthesized by PPC, all the macromolecules of the uniform sample have the same chain-length and sequence. This is convenient for some applications such as anti-counterfeiting and traceability technologies, in which a single type of sequence-coded chain is included and diluted in a host material. However, for other applications such as data storage or long-term archives, large quantities of data have to be stored and the storage capacity of a single polymer chain is certainly not sufficient. ${ }^{48}$ It is therefore important to study the 2D or 3D spatial organization of libraries of sequence-coded PPDE chains. This could be achieved by developing 2D micro-chips; a strategy that has already been explored for DNA data storage. ${ }^{49}$ In fact, common phosphoramidite-based strategies that are used for the preparation of DNA microchips, such as inkjet printing or photo-patterning, could all be applied to the 
fabrication of digital PPDE micro-arrays. However, this has not been achieved yet, even though the photo-controlled PPC of PPDE has already been validated. ${ }^{31}$ Beyond classical micro-chips, materials science offers other opportunities to organize polymers at the nanoscale. For instance, the spatial organization of PPDE libraries could be attained via co-crystallization, block copolymer self-assembly, ${ }^{50}$ DNA-guided self-assembly ${ }^{51}$ or LbL deposition. ${ }^{52}$ For instance, we have recently reported the LbL preparation of multilayered thin films containing segregated digital domains (Figure 5). ${ }^{53}$ In this approach, oppositely-charged polyelectrolytes are successively deposited on a planar substrate. Since digital PPDE are polyanions, they are ideal candidates for this technique. As a proof of principle, a 160-character citation of the French Chemist Marcellin Berthelot was decomposed into a library of sixteen different PPDE containing each 10 bytes of information. These polymers were then deposited stepwise on a modified silicon wafer. In order to achieve segregated digital domains, non-coded layers composed of commercial positively- and negatively-charged homopolymers were included between PPDE coded layers. The characterization of the modified surfaces indicated formation of $70 \mathrm{~nm}$-thick films, in which digital layers are kept apart from another by non-coded interlayers. The sequencing of such multilayered materials have not been reported yet and depth-profiling capabilities of DESI-MS/MS, recently shown to enable 2D-imaging of sequence-coded oligomers, ${ }^{54}$ are currently investigated. We have also reported very recently the DNA-guided self-organization of PPDE chains. ${ }^{55}$ Since PPC enables the use of both biological and non-biological monomers, bio-hybrid macromolecules containing long nonnatural segments covalently connected to single-stranded DNA terminal patches were prepared. Using complementary DNA strands, the precursors were then assembled by selective hybridization into linear of star-like superstructures. This proof-of-principle opens up interesting avenues for the design of informational networks or molecular circuitries based on digital PPDE. ${ }^{56}$ 


\section{OUTLOOK.}

After only five years of research, the design of abiological digital PPDE has drastically progressed. These synthetic informational polymers offer numerous advantages over natural nucleic acids for data storage and related applications. In terms of structural design, a storage density of 3 bits/monomer has already been reached with these polymers, which is above the one of natural DNA. This density is, moreover, probably not the highest that can be achieved. Furthermore, a single chain-capacity of 440 bits/chain has been attained, which is close to the one of DNA, at least when comparing chains obtained by chemical-synthesis. Significant progress has also been made regarding the MS/MS sequencing of these polymers and long information sequences can now be automatically decoded. Last but not least, it was shown that the information sequences of digital PPDE can be modified (i.e. erased, revealed or changed) by convenient external trigger, such as light exposure. Yet, there are still some aspects where these polymers are not as practical as DNA. For instance, the development of spatiallyorganized PPDE libraries is still in its infancy, even though interesting progress has been recently reported. Still, it shall be reminded that a very small amount of research has been conducted on this topic, in comparison to the very large efforts that have been dedicated to DNA nanotechnology over the last decades. Furthermore, since we focused intentionally on the design of single-stranded polymers, informational PPDE cannot be copied and amplified like natural nucleic acids. Nevertheless, this new family of informational macromolecules fills the gap between nucleic acids and conventional synthetic polymers. Apart from their relevance for several technological applications, these polymers may also be interesting models for understanding Life and developing xenobiological systems. ${ }^{57,58}$ Thus, the development of informational PPDE is expected to progress even further for the years to come. 


\section{FUNDING SOURCES}

The authors thank the French National Research Agency for the funded projects 00111001 (grant numbers ANR-16-CE29-0004-01 and ANR-16-CE29-0004-02), digital-LbL (grant numbers ANR-19-CE06-0020-01 and ANR-19-CE06-0020-02) and shapeNread (grant numbers ANR-19-CE29-0015-01 and ANR-19-CE29-0015-02).

\section{ACKNOWLEDGMENT}

J.-F.L. thanks Abdelaziz Al Ouahabi, Niklas Felix König, Eline Laurent, Tathagata Mondal, Maria Nerantzaki, Ian Roszak, Laurence Oswald, Thibault Schutz, Roza Szweda, Sofia Telitel and Yankai Zhang for their experimental contribution to this work. L.C. thanks Jean-Arthur Amalian for his involvement in ESI and DESI MS experiments. The authors also thank Alexandre Burel and Christine Carapito (IPHC, Strasbourg), Marc-André Delsuc (IGBMC, Strasbourg), Gero Decher and Olivier Felix (Institut Charles Sadron, Strasbourg), as well as Didier Gigmes and Jean-Louis Clément (Université Aix-Marseille) for fruitful collaborations. In addition, J.-F.L. also acknowledges successful collaborations with Jan Behrends (AlbertsLudwig-Universität, Freiburg), Cao Chan, Aleksandra Radenovic and Matteo Dal Peraro (EPFL, Lausanne), Yidan Cong and Sergei Sheiko (University of North Carolina, Chapel Hill) and Mounir Maaloum (Institut Charles Sadron, Strasbourg).

\section{ABBREVIATIONS}

CID, collision-induced dissociation; CuAAC, copper-catalyzed azide-alkyne cycloaddition; DESI, desorption electrospray ionization; DMT, 4,4'-dimethoxytrityl; ESI, electrospray ionization; LbL, Layer-bylayer; NPPOC, 2-(2-nitrophenyl)propoxycarbonyl; MS/MS, tandem mass spectrometry; PPC, phosphoramidite polymer chemistry; PPDE, poly(phosphodiester)s; RISC, Ring InSide Chain, TIPS, triisopropylsilyl. 

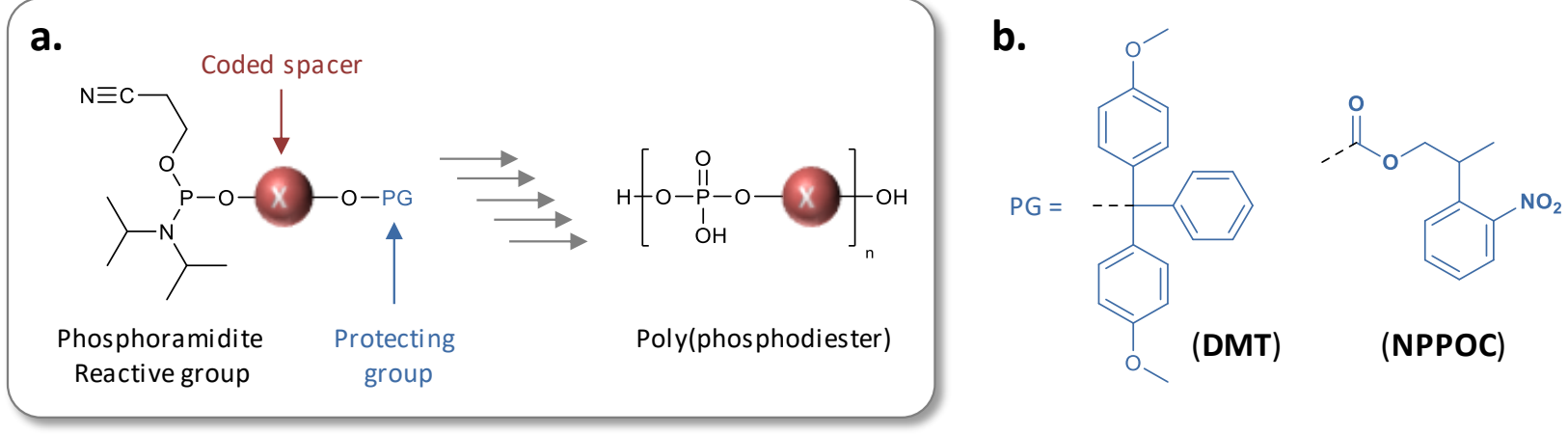

c.

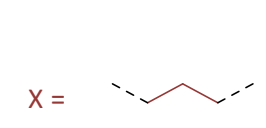

$\left(M_{1}\right)$

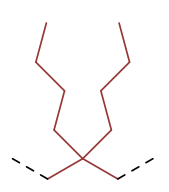

$\left(\mathrm{M}_{8}\right)$

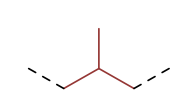

$\left(\mathrm{M}_{2}\right)$

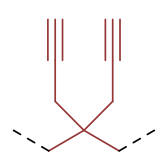

( $\left.\mathbf{M}_{\mathbf{g}}\right)$

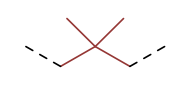

$\left(\mathbf{M}_{3}\right)$

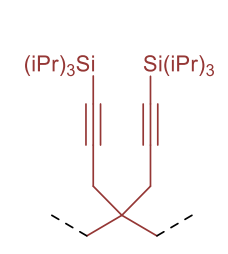

$\left(\mathrm{M}_{10}\right)$

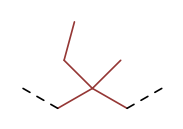

$\left(\mathrm{M}_{4}\right)$

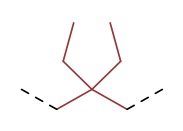

$\left(M_{5}\right)$

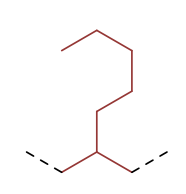

(M)

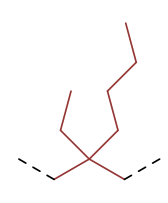

$\left(M_{7}\right)$

Figure 1. Molecular structure of the abiotic building blocks used for the construction of digital PPDE. (a) General molecular structure of a phosphoramidite monomer and of the resulting polymer. (b) Common protecting groups that are used in PPC. (c) Coded moieties that have been explored so far for the preparation of digital polymers. 
a.

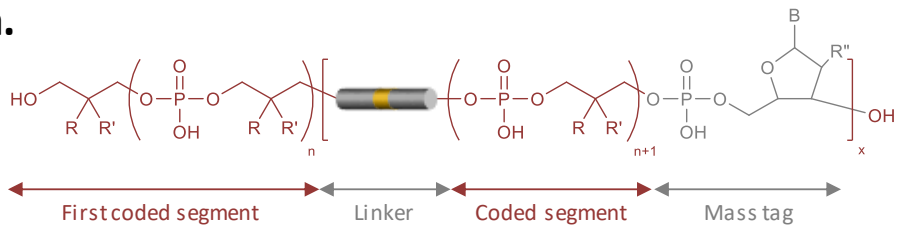

b.

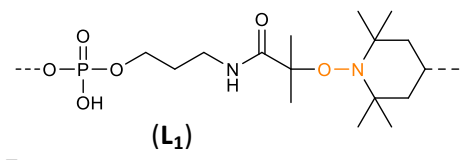

$\left(L_{1}\right)$

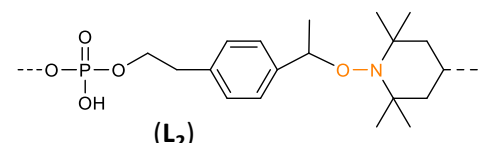

c.

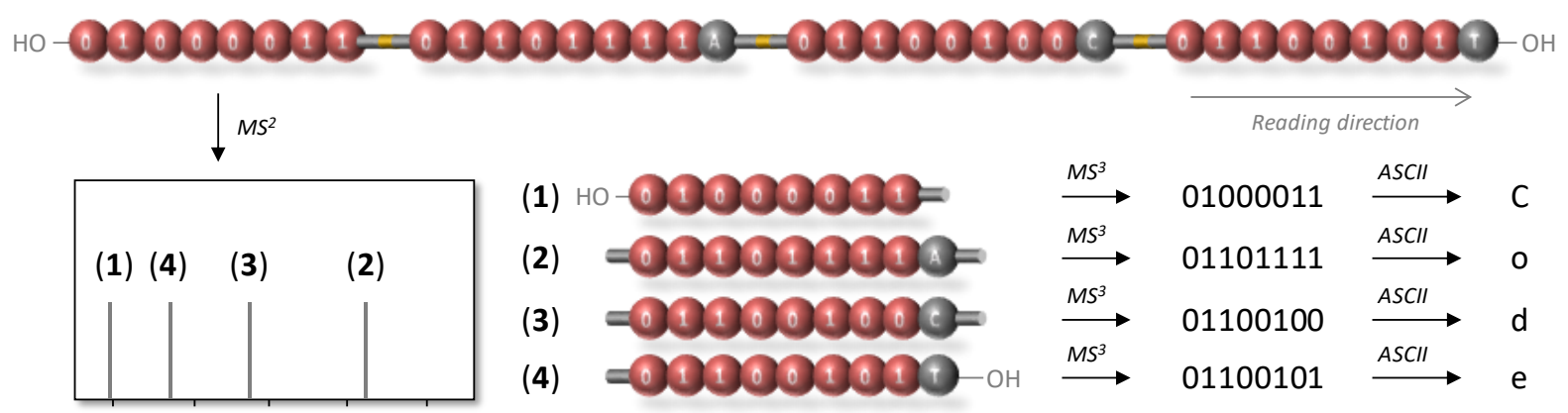

Figure 2. General concept for the sequencing of long digital PPDE. ${ }^{2}$ (a) Molecular structure of the polymers. They contain $x+1$ coded segments depicted in red. A fragment is a sequence of $n$ coded monomers $(n=8$ in most studies). Two consecutive fragments are separated by an alkoxyamine linker symbolized by a grey-yellow cylinder. In order to sort out the fragments after MS/MS cleavage, $x$ fragments of the sequence are labelled with a mass tag represented in grey. (b) Some alkoxyamine linkers that have been investigated in this concept. (c) Schematic representation of the concept. The digital polymer is first analyzed by MS/MS, which leads to the favored cleavage of the NO-C bonds (depicted in yellow). Since they carry mass tags (grey balls), the resulting fragments are sorted out by mass in the MS/MS spectrum. Afterwards, each byte can be sequenced in pseudo$\mathrm{MS}^{3}$ conditions and the whole binary sequence can be reconstructed. 

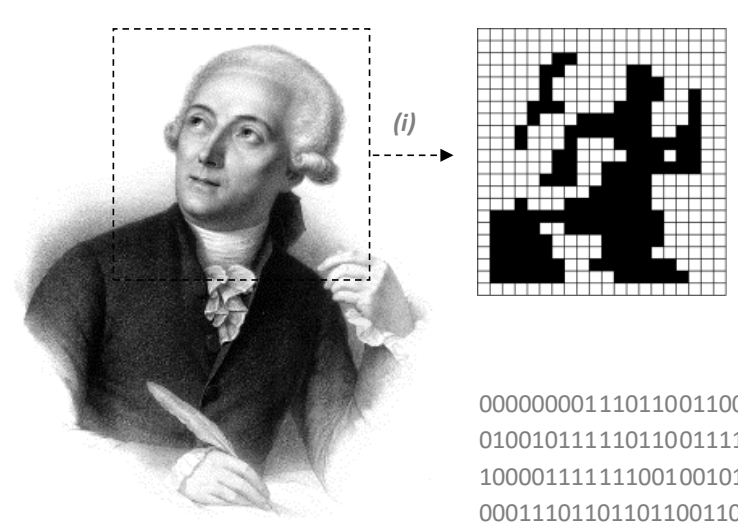

0000000000000000000000000000000000000000000000110000000 0000000000110000011000000000001000000111000000000010000 0011100100000011100001110001000000100011111100010000010 0011111110011000001000100011111110000000011000011101100 000000110011111110000000111001111000000000000000111110 0000000010001111111000000011111111111111000000111100111 1111100000011111000011111000000111110000111100000001111 1100111111100000111111000011111000000000000000000000000

(iii) Data compression

00000000111011001100111111101001101111111001100011001100111011010010110011110100 01001011111011001111011010010010101101000001011000110011101000010111111010001110 10000111111100100101111000001111000111110001111010001011101000111111100111111011 000111011011011001101111

(iv) Polymer encryption

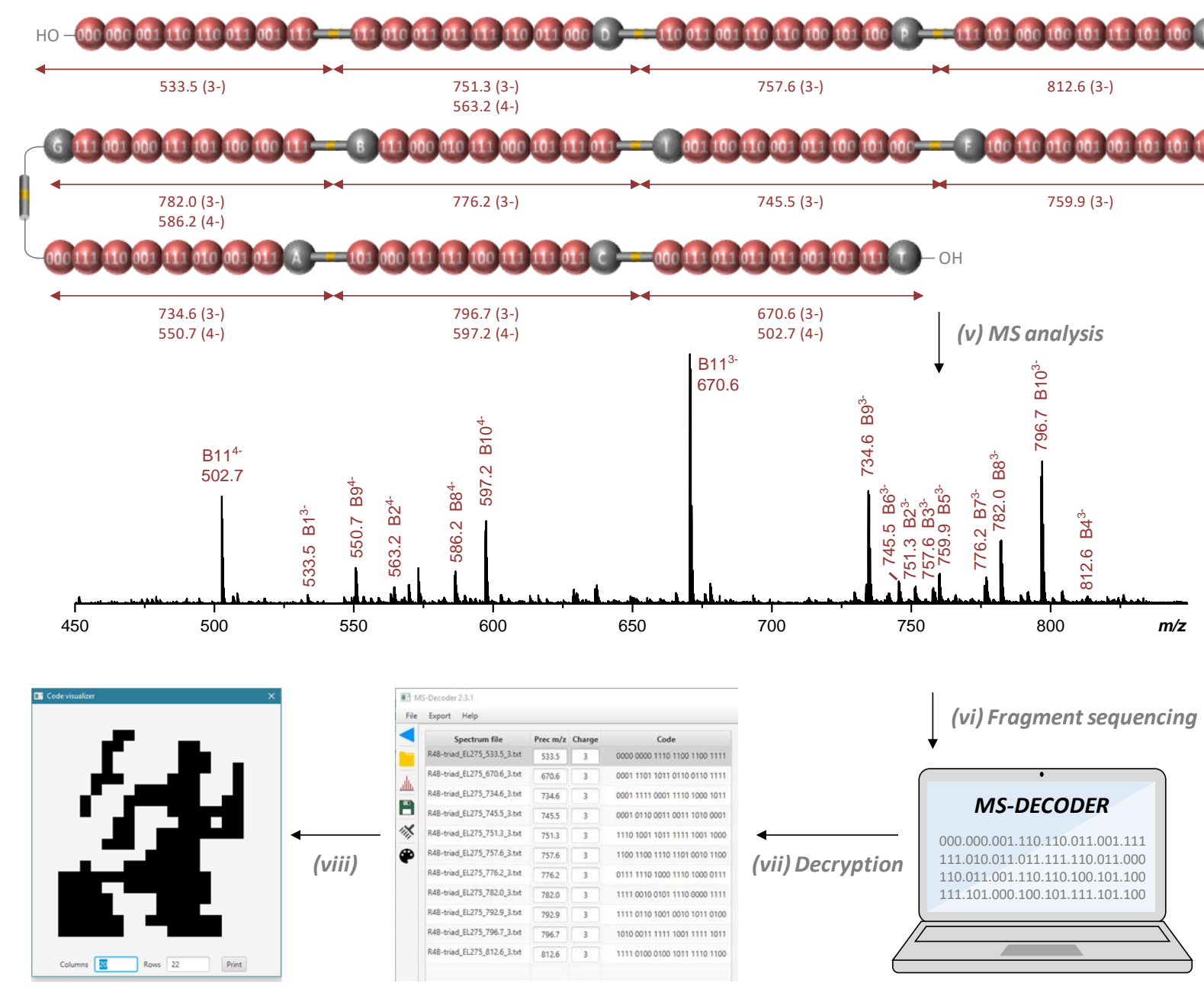

Figure 3. Encryption and automated decryption of a high-capacity digital PPDE. ${ }^{47}$ (i) The portrait of Antoine de Lavoisier (Author: François Séraphin Delpech, public domain, https://commons.wikimedia.org/wiki/File:Antoine_Laurent_de_Lavoisier.png) was first pixelated into a 20x22 image (440 pixels). (ii) The pixels were then transformed into a 440-bits string with 0 and 1 coding for white and black pixels, respectively. (iii) This digital sequence was then compressed into a 264-bits string. (iv) The compressed sequence was then translated into a chemical monomer sequence using monomers $\mathbf{M}_{\mathbf{1}}-\mathbf{M}_{\mathbf{8}}$, linker $\mathbf{L}_{\mathbf{2}}$ and appropriate mass tags. ( $v-v i)$ The PPDE is then analyzed by mass spectrometry and subjected to stepwise CID fragmentation. (vii) The spectra are decoded by the software MS-DECODER. (viii) Ultimately, information is decompressed and the pixelated image can be visualized. 
a.

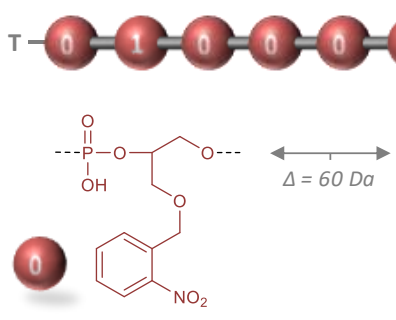

289.0 Da

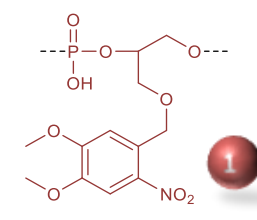

$349.1 \mathrm{Da}$

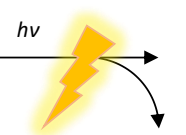<smiles>COc1cc(C=O)c([N+](=O)[O-])cc1OC</smiles>

$+$<smiles>O=Cc1ccccc1[N+](=O)[O-]</smiles>

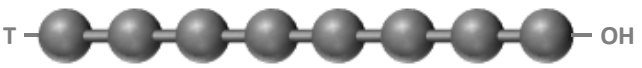<smiles>C[R](C)(C)O[R6](C)(=O)(O)OP(C)(=O)O</smiles>

b.
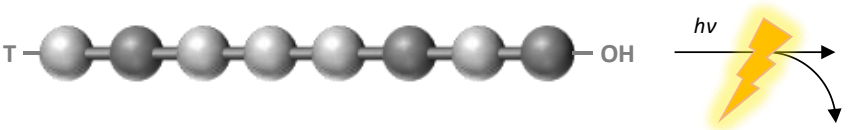<smiles>CC(C)(C)OC(COCc1ccccc1)COCC1[C@H]2C[C@H]1C2(C)C</smiles>

$289.0 \mathrm{Da}$

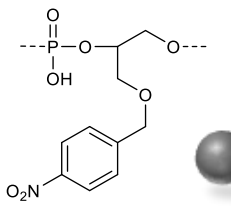

289.0 Da<smiles>O=Cc1ccccc1O</smiles>

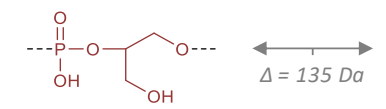

(0)

154.0 Da
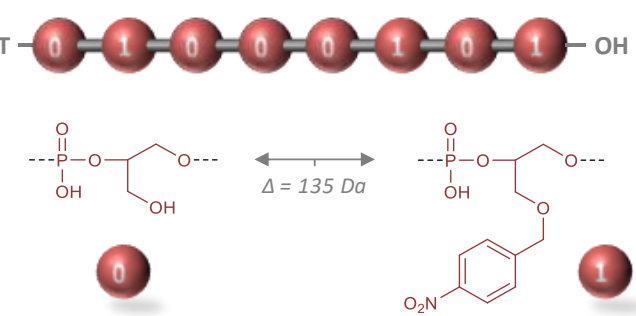

$289.0 \mathrm{Da}$

c.

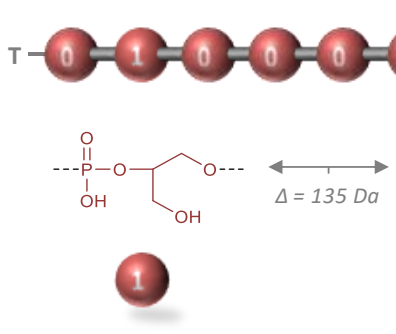

154.0 Da

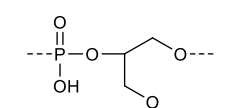<smiles>[R]c1ccc(CC)c([R])c1</smiles>

$289.0 \mathrm{Da}$

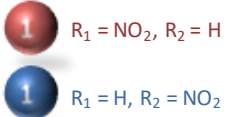
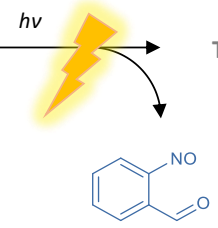

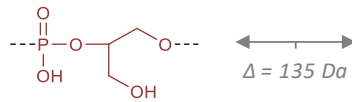

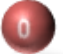

154.0 Da
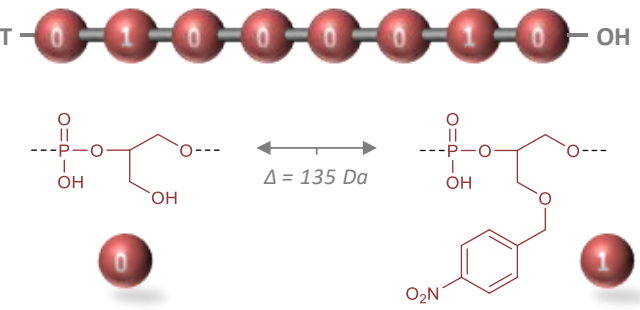

$289.0 \mathrm{Da}$

Figure 4. Examples of light-sensitive digital PPDE. ${ }^{3}$ (a) Photo-erasable digital polymers. Monomers $\mathbf{M}_{11}$ and $\mathbf{M}_{12}$ are used as 0 - and 1-bits, respectively. These monomers allow construction of digital sequences that can be decoded by MS/MS. Upon light irradiation, both 0 and 1 units are photo-cleaved, thus leading to a non-decodable homopolymer (depicted in grey). (b) Polymers containing hidden messages. Isobaric monomers $\mathbf{M}_{11}$ and $\mathbf{M}_{13}$ are used as an invisible binary ink, which lead to MS/MS non-decodable sequences. Light is then used as a revealer. Photo-irradiation cleaves selectively ortho-nitrobenzyl units, thus leading to a MS/MS decodable binary sequence. (c) Photo-induced site-directed mutations. The polymers are constructed using monomers $\mathbf{M}_{11}, \mathbf{M}_{13}$ and $\mathbf{M}_{\mathbf{1 4}}$. After triisopropyl silyl deprotection, the formed digital polymer contains isobaric 1-bits (depicted in red and blue). Light irradiation allows selective $1 \rightarrow 0$ mutation of a single type of 1 unit (i.e. only the blue one). The letter $\mathrm{T}$ symbolizes a terminal thymidine nucleoside. 


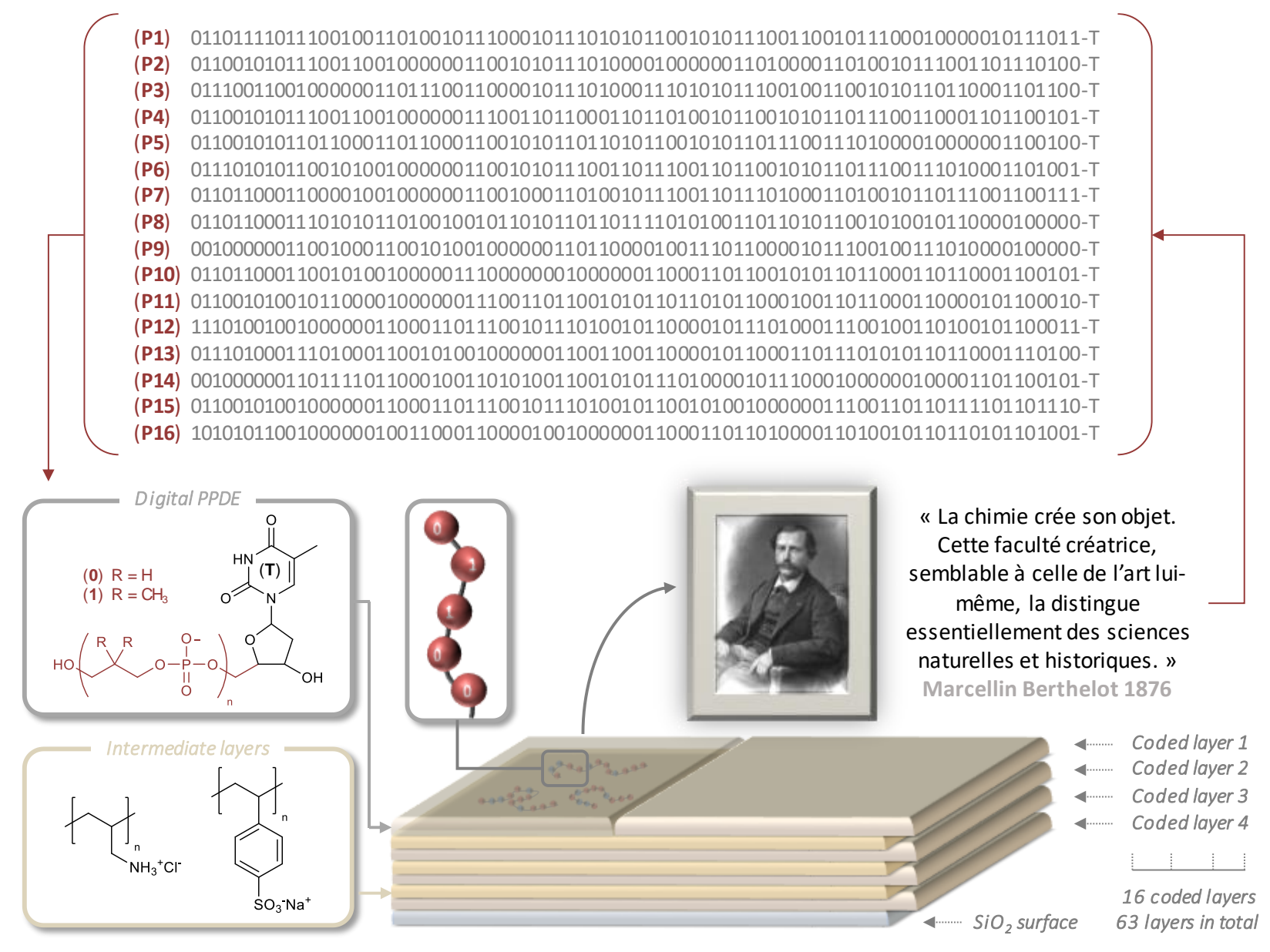

Figure 5. Preparation of a multilayered film containing digital information. ${ }^{53}$ In this approach, a citation of the French chemist Marcellin Berthelot was transformed into a binary text using standard ASCII rules. The text was fragmented into a library of 16 digitally-encoded PPDE (P1-P16). A multilayer film was then formed by LbL successive deposition of polyanions and polycations. The PPDE polyanions were deposited in a defined order and additional polyanion and polycation homopolymers were used to create intermediate layers of defined thickness. Consequently, each PPDE is entrapped in a segregated layer. The portrait of Marcellin Berthelot is in the public domain: https://commons.wikimedia.org/wiki/File:Marcellin_Berthelot_2.jpg. 


\section{REFERENCES}

(1) Al Ouahabi, A.; Charles, L.; Lutz, J.-F. Synthesis of Non-Natural Sequence-Encoded Polymers Using Phosphoramidite Chemistry. J. Am. Chem. Soc. 2015, 137, 5629-5635.

(2) Al Ouahabi, A.; Amalian, J.-A.; Charles, L.; Lutz, J.-F. Mass spectrometry sequencing of long digital polymers facilitated by programmed inter-byte fragmentation. Nat. Commun. 2017, 8, 967.

(3) König, N. F.; Al Ouahabi, A.; Oswald, L.; Szweda, R.; Charles, L.; Lutz, J.-F. Photoeditable macromolecular information. Nat. Commun. 2019, 10, 3774.

(4) Laurent, E.; Amalian, J.-A.; Parmentier, M.; Oswald, L.; Al Ouahabi, A.; Dufour, F.; Launay, K.; Clément, J.-L.; Gigmes, D.; Delsuc, M.-A.; Charles, L.; Lutz, J.-F. HighCapacity Digital Polymers: Storing Images in Single Molecules. Macromolecules 2020, 53, 4022-4029.

(5) Lutz, J.-F. Writing on Polymer Chains. Acc. Chem. Res. 2013, 46, 2696-2705.

(6) Lutz, J.-F.; Ouchi, M.; Liu, D. R.; Sawamoto, M. Sequence-Controlled Polymers. Science 2013, 341, 1238149.

(7) Lutz, J.-F. Defining the Field of Sequence-Controlled Polymers. Macromol. Rapid Commun. 2017, 38, 1700582.

(8) Szymański, J. K.; Abul-Haija, Y. M.; Cronin, L. Exploring Strategies To Bias Sequence in Natural and Synthetic Oligomers and Polymers. Acc. Chem. Res. 2018, 51, 649-658.

(9) Trinh, T. T.; Oswald, L.; Chan-Seng, D.; Lutz, J.-F. Synthesis of Molecularly Encoded Oligomers Using a Chemoselective "AB + CD" Iterative Approach. Macromol. Rapid Commun. 2014, 35, 141-145.

(10) Roy, R. K.; Meszynska, A.; Laure, C.; Charles, L.; Verchin, C.; Lutz, J.-F. Design and Synthesis of Digitally-Encoded Polymers that can be Decoded and Erased. Nat. Commun. 2015, 6, 7237.

(11) Gunay, U. S.; Petit, B. E.; Karamessini, D.; Al Ouahabi, A.; Amalian, J.-A.; Chendo, C.; Bouquey, M.; Gigmes, D.; Charles, L.; Lutz, J.-F. Chemoselective Synthesis of Uniform Sequence-Coded Polyurethanes and Their Use as Molecular Tags. Chem 2016, $1,114-126$.

(12) Cavallo, G.; Al Ouahabi, A.; Oswald, L.; Charles, L.; Lutz, J.-F. Orthogonal Synthesis of "Easy-to-Read" Information-Containing Polymers Using Phosphoramidite and Radical Coupling Steps. J. Am. Chem. Soc. 2016, 138, 9417-9420.

(13) Boukis, A. C.; Meier, M. A. R. Data storage in sequence-defined macromolecules via multicomponent reactions. Eur. Polym. J. 2018, 104, 32-38.

(14) Martens, S.; Landuyt, A.; Espeel, P.; Devreese, B.; Dawyndt, P.; Du Prez, F. Multifunctional sequence-defined macromolecules for chemical data storage. Nat. Commun. 2018, 9, 4451. 
(15) Huang, Z.; Shi, Q.; Guo, J.; Meng, F.; Zhang, Y.; Lu, Y.; Qian, Z.; Li, X.; Zhou, N.; Zhang, Z.; Zhu, X. Binary tree-inspired digital dendrimer. Nat. Commun. 2019, 10, 1918.

(16) Lee, J. M.; Koo, M. B.; Lee, S. W.; Lee, H.; Kwon, J.; Shim, Y. H.; Kim, S. Y.; Kim, $\mathrm{K}$. T. High-density information storage in an absolutely defined aperiodic sequence of monodisperse copolyester. Nat. Commun. 2020, 11, 56.

(17) Colquhoun, H.; Lutz, J.-F. Information-containing macromolecules. Nat. Chem. 2014, $6,455-456$.

(18) Rutten, M. G. T. A.; Vaandrager, F. W.; Elemans, J. A. A. W.; Nolte, R. J. M. Encoding information into polymers. Nat. Rev. Chem. 2018, 2, 365-381.

(19) Lutz, J.-F. Coding Macromolecules: Inputting Information in Polymers Using Monomer-Based Alphabets. Macromolecules 2015, 48, 4759-4767.

(20) Lutz, J.-F.; Lehn, J.-M.; Meijer, E. W.; Matyjaszewski, K. From Precision Polymers to Complex Materials and Systems. Nat. Rev. Mater. 2016, 1, 16024.

(21) Mutlu, H.; Lutz, J.-F. Reading Polymers: Sequencing of Natural and Synthetic Macromolecules. Angew. Chem., Int. Ed. 2014, 53, 13010-13019.

(22) Shendure, J.; Balasubramanian, S.; Church, G. M.; Gilbert, W.; Rogers, J.; Schloss, J. A.; Waterston, R. H. DNA sequencing at 40: past, present and future. Nature 2017, 550, 345-353.

(23) Wesdemiotis, C. Multidimensional Mass Spectrometry of Synthetic Polymers and Advanced Materials. Angew. Chem., Int. Ed. 2017, 56, 1452-1464.

(24) Branton, D.; Deamer, D. W.; Marziali, A.; Bayley, H.; Benner, S. A.; Butler, T.; Di Ventra, M.; Garaj, S.; Hibbs, A.; Huang, X.; Jovanovich, S. B.; Krstic, P. S.; Lindsay, S.; Ling, X. S.; Mastrangelo, C. H.; Meller, A.; Oliver, J. S.; Pershin, Y. V.; Ramsey, J. M.; Riehn, R.; Soni, G. V.; Tabard-Cossa, V.; Wanunu, M.; Wiggin, M.; Schloss, J. A. The potential and challenges of nanopore sequencing. Nat. Biotech. 2008, 26, 11461153.

(25) Beaucage, S. L.; Iyer, R. P. Advances in the Synthesis of Oligonucleotides by the Phosphoramidite Approach. Tetrahedron 1992, 48, 2223-2311.

(26) Appukutti, N.; Serpell, C. J. High definition polyphosphoesters: between nucleic acids and plastics. Polym. Chem. 2018, 9, 2210-2226.

(27) Vybornyi, M.; Vyborna, Y.; Häner, R. DNA-inspired oligomers: from oligophosphates to functional materials. Chem. Soc. Rev. 2019, 48, 4347-4360.

(28) Tost, J.; Gut, I. G. DNA analysis by mass spectrometry-past, present and future. $J$. Mass Spectrom. 2006, 41, 981-995.

(29) Beaucage, S. L.; Caruthers, M. H. Deoxynucleoside phosphoramidites-A new class of key intermediates for deoxypolynucleotide synthesis. Tetrahedron Lett. 1981, 22, 1859-1862. 
(30) Hasan, A.; Stengele, K.-P.; Giegrich, H.; Cornwell, P.; Isham, K. R.; Sachleben, R. A.; Pfleiderer, W.; Foote, R. S. Photolabile protecting groups for nucleosides: Synthesis and photodeprotection rates. Tetrahedron 1997, 53, 4247-4264.

(31) König, N. F.; Telitel, S.; Poyer, S.; Charles, L.; Lutz, J.-F. Photocontrolled Synthesis of Abiotic Sequence-Defined Oligo(Phosphodiester)s. Macromol. Rapid Commun. 2017, $38,1700651$.

(32) Caruthers, M. H. Gene synthesis machines: DNA chemistry and its uses. Science 1985, 230, 281-285.

(33) Schnitzler, T.; Herrmann, A. DNA Block Copolymers: Functional Materials for Nanoscience and Biomedicine. Acc. Chem. Res. 2012, 45, 1419-1430.

(34) Edwardson, T. G. W.; Carneiro, K. M. M.; Serpell, C. J.; Sleiman, H. F. An Efficient and Modular Route to Sequence-Defined Polymers Appended to DNA. Angew. Chem., Int. Ed. 2014, 53, 4567-4571.

Appukutti, N.; Jones, J. R.; Serpell, C. J. Sequence isomerism in uniform polyphosphoesters programmes self-assembly and folding. Chem. Commun. 2020, 56, 5307-5310.

(36) Al Ouahabi, A.; Kotera, M.; Charles, L.; Lutz, J.-F. Synthesis of Monodisperse Sequence-Coded Polymers with Chain Lengths above DP100. ACS Macro Lett. 2015, 4, 1077-1080.

(37) Amalian, J. A.; Al Ouahabi, A.; Cavallo, G.; König, N. F.; Poyer, S.; Lutz, J. F.; Charles, L. Controlling the structure of sequence-defined poly(phosphodiester)s for optimal MS/MS reading of digital information. J. Mass Spectrom. 2017, 52, 788-798.

(38) Cao, C.; Krapp, L. F.; Al Ouahabi, A.; König, N. F.; Cirauqui, N.; Radenovic, A.; Lutz, J.-F.; Peraro, M. D. Aerolysin nanopores decode digital information stored in tailored macromolecular analytes. Sci. Adv. 2020, 6, eabc2661.

(39) König, N. F.; Al Ouahabi, A.; Poyer, S.; Charles, L.; Lutz, J.-F. A Simple PostPolymerization Modification Method for Controlling Side-Chain Information in Digital Polymers. Angew. Chem., Int. Ed. 2017, 56, 7297-7301.

(40) Safak, M.; Alemdaroglu, F. E.; Li, Y.; Ergen, E.; Herrmann, A. Polymerase Chain Reaction as an Efficient Tool for the Preparation of Block Copolymers. Adv. Mater. 2007, 19, 1499-1505.

(41) Alemdaroglu, F. E.; Zhuang, W.; Zöphel, L.; Wang, J.; Berger, R.; Rabe, J. P.; Herrmann, A. Generation of Multiblock Copolymers by PCR: Synthesis, Visualization and Nanomechanical Properties. Nano Lett. 2009, 9, 3658-3662.

(42) Boukhet, M.; König, N. F.; Ouahabi, A. A.; Baaken, G.; Lutz, J.-F.; Behrends, J. C. Translocation of Precision Polymers through Biological Nanopores. Macromol. Rapid Commun. 2017, 38, 1700680.

(43) Nordhoff, E.; Kirpekar, F.; Roepstorff, P. Mass spectrometry of nucleic acids. Mass Spectrom. Rev. 1996, 15, 67-138. 
(44) Audran, G.; Marque, S. R. A.; Mellet, P. Smart Alkoxyamines: A New Tool for Smart Applications. Acc. Chem. Res. 2020, 53, 2828-2840.

(45) Burel, A.; Carapito, C.; Lutz, J.-F.; Charles, L. MS-DECODER: Milliseconds Sequencing of Coded Polymers. Macromolecules 2017, 50, 8290-8296.

(46) Launay, K.; Amalian, J.-A.; Laurent, E.; Oswald, L.; Al Ouahabi, A.; Burel, A.; Dufour, F.; Carapito, C.; Clément, J.-L.; Lutz, J.-F.; Charles, L.; Gigmes, D. Precise Alkoxyamine Design to Enable Automated Tandem Mass Spectrometry Sequencing of Digital Poly(phosphodiester)s. Angew. Chem., Int. Ed. 2021, 60, 917-926.

(47) Laurent, E.; Amalian, J. A.; Schutz, T.; Launay, K.; Clément, J.-L.; Gigmes, D.; Burel, A.; Carapito, C.; Charles, L.; Delsuc, M.-A.; Lutz, J. F. Storing the portrait of Antoine de Lavoisier in a Single Macromolecule. $C . R$. Chim., in press. https://doi.org/10.5802/crchim.72.

(48) Steinkoenig, J.; Aksakal, R.; Du Prez, F. Molecular access to multi-dimensionally encoded information. Eur. Polym. J. 2019, 120, 109260.

(49) Ceze, L.; Nivala, J.; Strauss, K. Molecular digital data storage using DNA. Nat. Rev. Genet. 2019, 20, 456-466.

(50) Albert, J. N. L.; Epps, T. H. Self-assembly of block copolymer thin films. Mater. Today 2010, 13, 24-33.

(51) Aldaye, F. A.; Palmer, A. L.; Sleiman, H. F. Assembling Materials with DNA as the Guide. Science 2008, 321, 1795.

(52) Decher, G. Fuzzy Nanoassemblies: Toward Layered Polymeric Multicomposites. Science 1997, 277, 1232.

(53) Szweda, R.; Tschopp, M.; Felix, O.; Decher, G.; Lutz, J.-F. Sequences of Sequences: Spatial Organization of Coded Matter through Layer-by-Layer Assembly of Digital Polymers. Angew. Chem., Int. Ed. 2018, 57, 15817-15821.

(54) Amalian, J. A.; Mondal, T.; Konishcheva, E.; Cavallo, G.; Petit, B. E.; Lutz, J. F.; Charles, L. Desorption electrospray ionization (DESI) of digital polymers: direct tandem mass spectrometry decoding and imaging from materials surfaces. Adv. Mater. Technol. 2021, 6, 2001088.

(55) Mondal, T.; Nerantzaki, M.; Maaloum, M.; Cong, Y.; Sheiko, S. S.; Lutz, J.-F. Large sequence-defined supramolecules obtained by DNA-guided assembly of bio-hybrid poly(phosphodiester)s. Submitted to Macromolecules.

(56) LaBoda, C.; Duschl, H.; Dwyer, C. L. DNA-Enabled Integrated Molecular Systems for Computation and Sensing. Acc. Chem. Res. 2014, 47, 1816-1824.

(57) Lutz, J.-F. Can Life Emerge from Synthetic Polymers? Isr. J. Chem. 2020, 60, 151-159.

(58) Lutz, J.-F. 100th Anniversary of Macromolecular Science Viewpoint: Toward Artificial Life-Supporting Macromolecules. ACS Macro Lett. 2020, 9, 185-189. 